\title{
Supervised Self-Organizing Classification of Superresolution ISAR Images: An Anechoic Chamber Experiment
}

\author{
Emanuel Radoi, André Quinquis, and Felix Totir \\ ENSIETA, E3I2 Research Center, 2 rue François Verny, 29806 Brest, France \\ Received 1 June 2005; Revised 30 January 2006; Accepted 5 February 2006
}

\begin{abstract}
The problem of the automatic classification of superresolution ISAR images is addressed in the paper. We describe an anechoic chamber experiment involving ten-scale-reduced aircraft models. The radar images of these targets are reconstructed using MUSIC-2D (multiple signal classification) method coupled with two additional processing steps: phase unwrapping and symmetry enhancement. A feature vector is then proposed including Fourier descriptors and moment invariants, which are calculated from the target shape and the scattering center distribution extracted from each reconstructed image. The classification is finally performed by a new self-organizing neural network called SART (supervised ART), which is compared to two standard classifiers, MLP (multilayer perceptron) and fuzzy KNN ( $K$ nearest neighbors). While the classification accuracy is similar, SART is shown to outperform the two other classifiers in terms of training speed and classification speed, especially for large databases. It is also easier to use since it does not require any input parameter related to its structure.
\end{abstract}

Copyright (C) 2006 Emanuel Radoi et al. This is an open access article distributed under the Creative Commons Attribution License, which permits unrestricted use, distribution, and reproduction in any medium, provided the original work is properly cited.

\section{INTRODUCTION}

Our research work has been focused for several years on ISAR techniques and automatic target recognition (ATR) using superresolution radar imagery. The anechoic chamber of ENSIETA and the associated measurement facilities allow us to obtain radar signatures for various scale-reduced targets and to reconstruct their radar images using a turntable configuration. The main advantage of this type of configuration is the capability to achieve realistic measurements, to have a perfect control of the target configuration, and to simplify the interpretation of the obtained results.

We have already presented in [1] some of our significant results on both theoretical and practical aspects related to the application of superresolution imagery techniques. Since a critical point for the application of these methods is the estimation of the number of scattering centers (the same as the signal subspace dimension), we have also proposed in [2] a discriminative learning-based algorithm to perform this task.

The objective of this paper is to investigate another aspect, which is considered with increasing interest in the ATR field, that is, the automatic classification of ISAR images. This is a very challenging task for radar systems, which are generally designed to perform target detection and localization. The power of the backscattered signal, the receiver sensitivity, and the signal-to-noise ratio are determinants for detecting and localizing a radar target, but are much less important for classifying it. On the other hand, the information related to the target shape becomes essential whenever the goal is its classification [3]. Two conditions have to be met in order to define an imagery based-target classification procedure:

(1) the imaging method should be able to provide the information about the target shape with a maximum of accuracy;

(2) the classification technique should be able to exploit the information contained by the reconstructed image.

The accuracy of the target shape is closely related to the available spatial resolution. It is mainly given by the frequency bandwidth and the integration angle domain (spatial frequency bandwidth) when the Fourier transform is used for performing the imaging process [4]. Actually, the cross range resolution is limited by the integration time, which should be short enough to avoid image defocusing due to scattering center migration or nonuniform rotation motion [4]. Furthermore, the choice of the weighting window always requires a trade-off between the spatial and the dynamic resolutions.

For all these reasons we have decided to work with orthogonal subspace decomposition-based imaging techniques, which are able to provide high resolution, even for 
very limited angular domains and frequency bandwidths. These methods, also known as superresolution techniques, are mainly based on the eigenanalysis of the data covariance matrix and their use is advantageous especially for maneuvering or very mobile targets. One such method, called MUSIC-2D (multiple signal classification) [5], is used in this paper because of its effectiveness and robustness. Indeed, the maxima corresponding to the scattering centers are readily found by the projection of a mode vector onto the noise subspace. The algorithm is not very sensitive to the subspace dimension estimation, while a statistical analysis indicates performance close to the Cramer-Rao bound on location accuracy $[6]$.

The capability of the classifier to exploit the information in the reconstructed image is assessed primarily by the classification performance. The classifier performance level depends on both its structure and training process parameter choice. From this point of view, powerful nonlinear structures like neural networks or nonparametric methods are very attractive candidates to perform the classification. At the same time, the number of parameters required by the training process should be reduced as much as possible and their values should not be critical for obtaining the optimal solution.

Hence, another objective of the paper is to evaluate the performance of a classifier we have developed recently in the framework of ATR using feature vectors extracted from ISAR images. This classifier, called SART (supervised ART), has the structure of a self-organizing neural network and combines the principles of VQ (vector quantization) [7] and ART (adaptive resonance theory) [8]. The training algorithm requires only a few input parameters, whose values are not critical for the classifier performance. It converges very quickly and integrates effective rules for rejecting outliers.

The rest of the paper is organized as follows. The experiment setup and the principle of the imaging process are presented in Section 2. The extraction of the feature vector is explained through several examples in Section 3. SART classifier structure and training algorithm are introduced in Section 4, while Section 5 is devoted to the presentation and discussion of the classification results obtained using the proposed approach. Some conclusions are finally drawn in Section 6 together with some ideas and projections related to our future research work.

\section{EXPERIMENT DESCRIPTION AND IMAGE ACQUISITION}

The experimental setup is shown in Figure 1. The central part of the measurement system is the vector network analyzer (Wiltron 360) driven by a PC Pentium IV by means of a Labview 7.1 interface. The frequency synthesizer generates a frequency-stepped signal, whose frequency band can be chosen between $2 \mathrm{GHz}$ and $18 \mathrm{GHz}$. The frequency step value and number are set in order to obtain a given slant range resolution and slant range ambiguity window. The echo signal is passed through a low-noise amplifier (Miteq AMF-4D-
020180-23-10P) and then quadrature detection is used by the network analyzer to compute the complex target signature.

The ten targets used in our experiment are shown in Figure 2. They represent aircraft scale-reduced models (1 : 48 ) and are made of plastic with a metallic coating. These targets are placed on a dielectric turntable, which is rotated by a multiaxis positioning control system (Newport MM4006). It is also driven by the PC and provides a precision of $0.01^{\circ}$.

Each target is illuminated in the acquisition phase with a frequency-stepped signal. The data snapshot contains 31 frequency steps, uniformly distributed over the $\mathrm{Ku}$ band $\Delta f=(12,18) \mathrm{GHz}$, which results in a frequency increment $\delta f=200 \mathrm{MHz}$. The equivalent effective center frequency and bandwidth against full-scale targets are then obtained as 312.5 MHz and $125 \mathrm{MHz}$, respectively.

Ninety images of each target have been generated for aspect angles between $0^{\circ}$ and $90^{\circ}$, with an angular shift between two consecutive images of $1^{\circ}$. Each image is obtained from the complex signatures recorded over an angular sector of $10^{\circ}$, with an angular increment of $1^{\circ}$.

After data resampling and interpolation the following values are obtained for the slant range and cross range resolutions and ambiguity windows:

$$
\begin{aligned}
& \Delta R_{s} \cong 2.5 \mathrm{~cm}, \quad W_{s} \cong 0.75 \mathrm{~m}, \\
& \Delta R_{c} \cong 7.4 \mathrm{~cm}, \quad W_{c} \cong 0.74 \mathrm{~m} .
\end{aligned}
$$

The main steps involved in the radar target image reconstruction using MUSIC-2D method are given below [1]:

(1) 2D array complex data acquisition;

(2) data preprocessing using the polar formatting algorithm (PFA) [4];

(3) estimation of the autocorrelation matrix using the spatial smoothing method [9];

(4) eigenanalysis of the autocorrelation matrix and identification of the eigenvectors associated to the noise subspace using AIC or MDL method [10];

(5) MUSIC-2D reconstruction of the radar image by projecting the mode vector onto the noise subspace in each point of the data grid.

The flowchart of the superresolution imaging algorithm is shown in Figure 3. Each processing stage is illustrated with a generic example for a better understanding of the operations involved in the reconstruction process.

The main idea is to estimate the scattering center positions by searching the maxima of the function below, which is evaluated for a finite number of points $(x, y)$ :

$$
P_{\text {MUSiC-2D }}(x, y)=\frac{1}{\mathbf{a}(x, y)^{H} \mathbf{V}_{\mathbf{n}} \mathbf{V}_{\mathbf{n}}^{\mathbf{H}} \mathbf{a}(x, y)}
$$

In the equation above $\mathbf{V}_{\mathbf{n}}$ is the matrix whose columns are the eigenvectors corresponding to the noise subspace, 


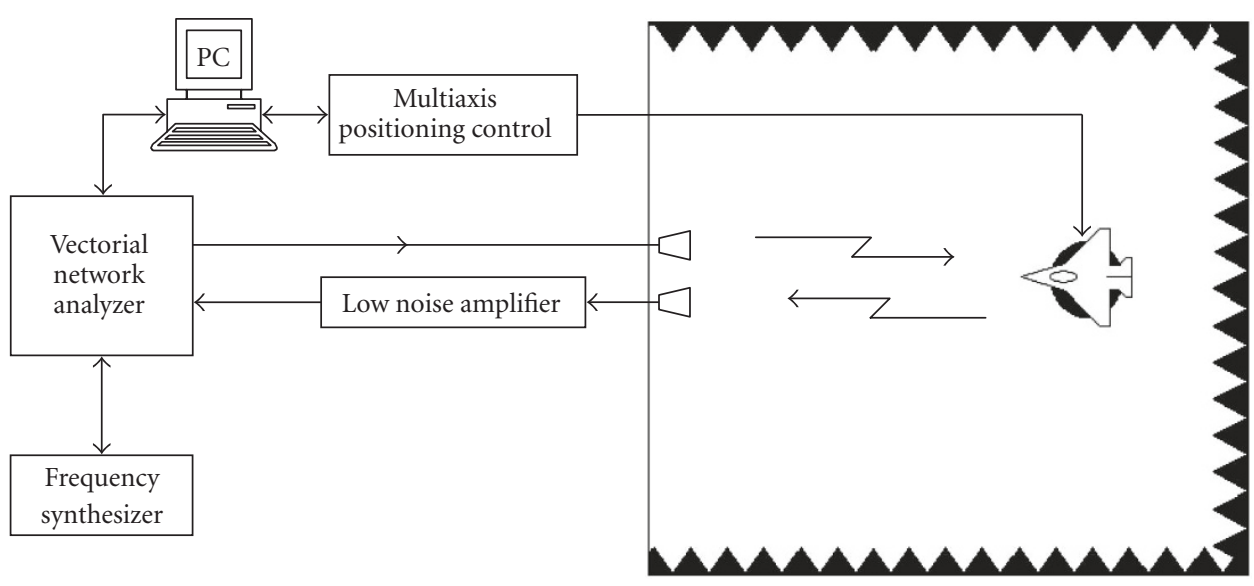

(a) Block diagram of the acquisition system

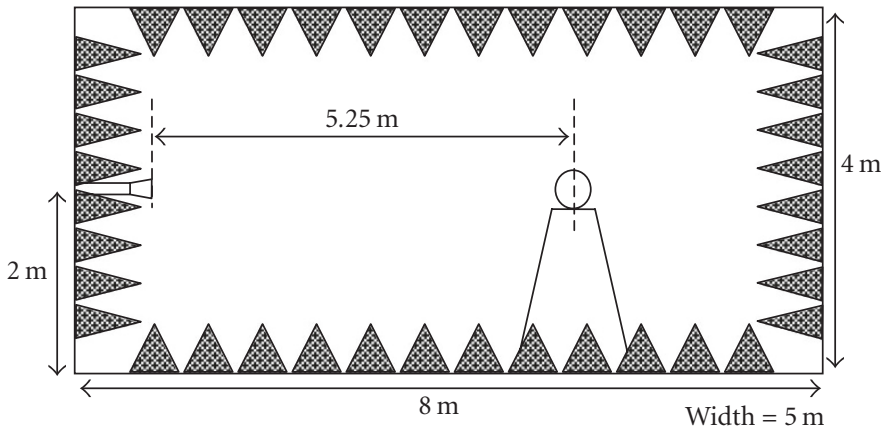

(b) Main dimensions of the anechoic chamber

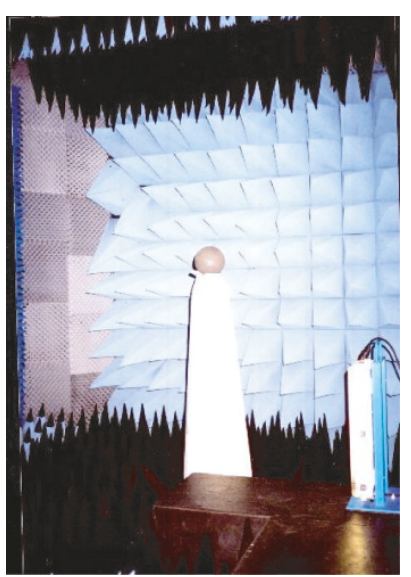

(c) Anechoic chamber inside

Figure 1: Measurement system configuration.

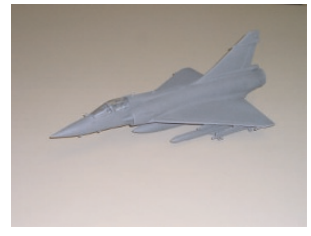

(a) Mirage

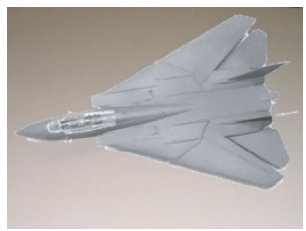

(f) F-14

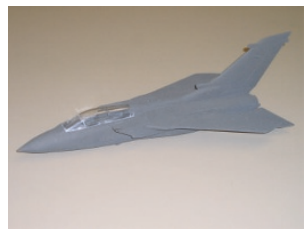

(b) Tornado

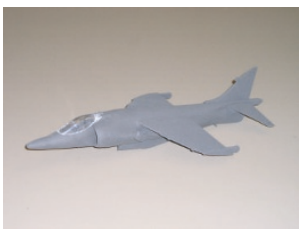

(g) Harrier

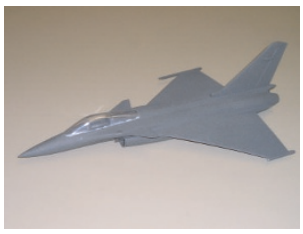

(c) Rafale

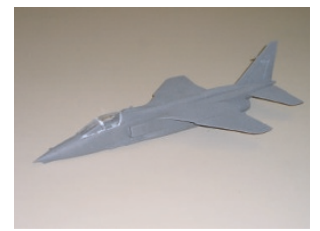

(h) Jaguar

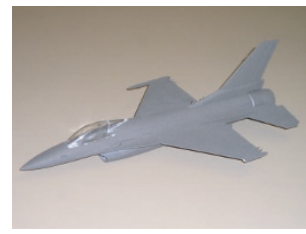

(d) F-16

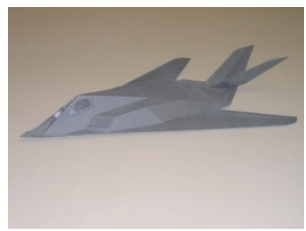

(i) F-117

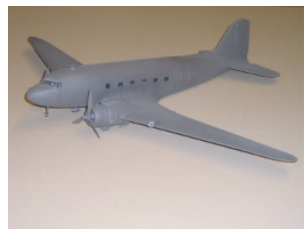

(e) DC-3

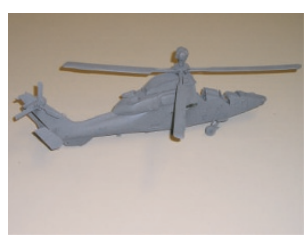

(j) AH-64

FIGURE 2: Scale-reduced aircraft models measured in the anechoic chamber. 

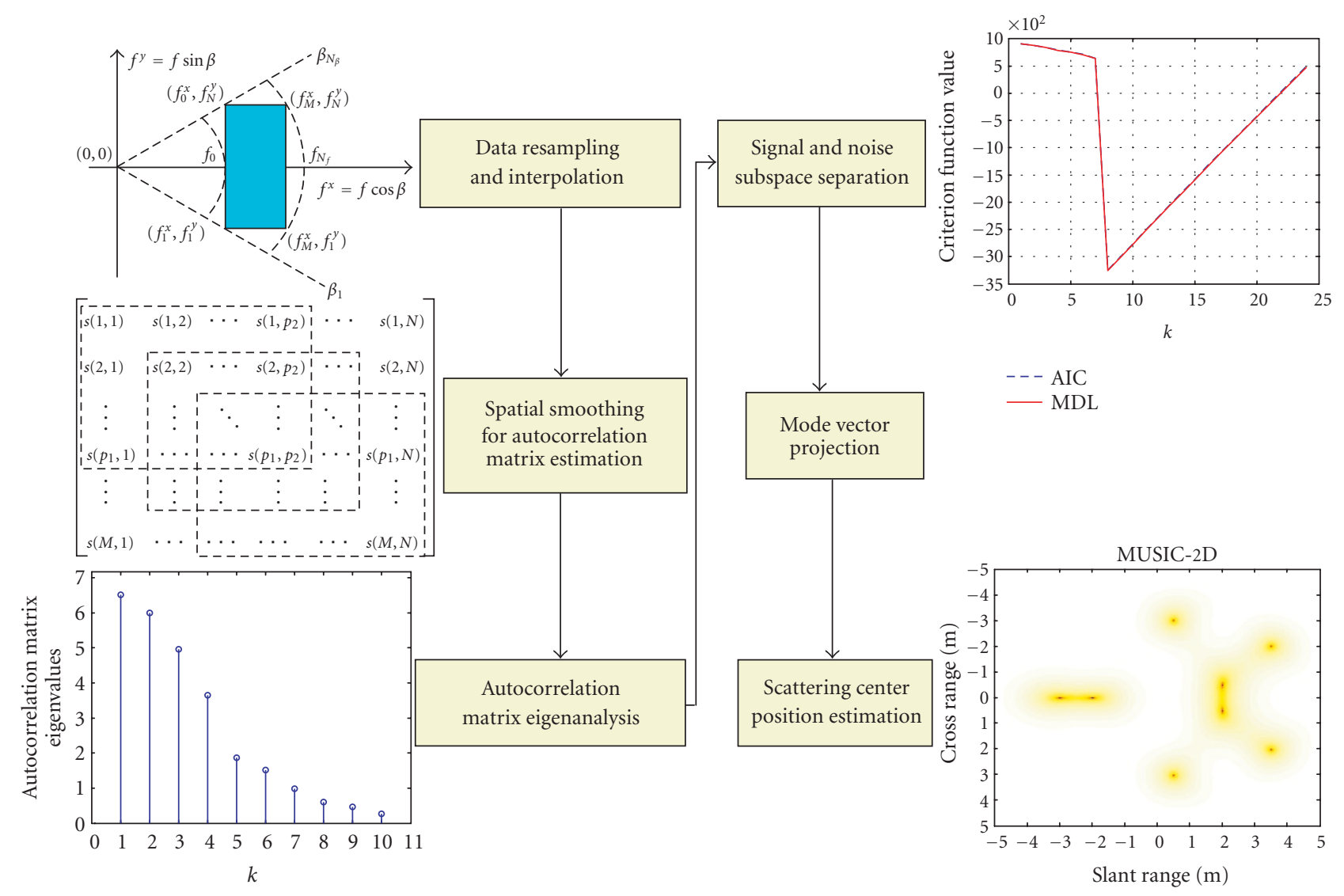

FigURE 3: Flowchart of the imaging process using MUSIC-2D method.

and $\mathbf{a}(x, y)$ stands for the mode vector:

$$
\begin{aligned}
\mathbf{a}(x, y)=[ & \exp \left(j \frac{4 \pi}{c}\left(f_{0}^{(x)} x+f_{0}^{(y)} y\right)\right) \cdots \\
& \quad \exp \left(j \frac{4 \pi}{c}\left(f_{N_{1}-1}^{(x)} x+f_{0}^{(y)} y\right)\right) \cdots \\
& \left.\exp \left(j \frac{4 \pi}{c}\left(f_{N_{1}-1}^{(x)} x+f_{N_{2}-1}^{(y)} y\right)\right)\right]^{T},
\end{aligned}
$$

where $f^{(x)}=f \cos \beta$ and $f^{(y)}=f \sin \beta$ define the Cartesian grid obtained after resampling the polar grid $(f, \beta)$ (frequency and azimuth angle), which is actually used for data acquisition.

\section{FEATURE VECTORS}

Two types of features, extracted from the reconstructed images, have been used in our experiment in order to obtain a good separation of the 10 classes. The feature extraction process is illustrated in Figure 4 for the case of the DC-3 aircraft, at $\beta=0^{\circ}$.
The image issued directly from the superresolution imaging algorithm is called "rough reconstructed image." A phase unwrapping algorithm [11] and a symmetry enhancement technique [12] are then applied in order to improve the quality of the reconstructed image and to make the extracted features more robust. In Figure 4 the image processed in this way is called "reconstructed image after phase correction."

Our hypothesis is that the information about the target type is mainly carried by its shape and scattering center distribution. The scattering centers are first extracted using a running mask of $3 \times 3$ pixels and a simple rule: a new scattering center is detected whenever the value of the pixel in the center of the mask is the largest compared to its neighbors. The target shape is then extracted using active deformable contours or "snakes" [13]. They are edge-attracted, elastic evolving shapes, which iteratively reach a final position, representing a trade-off between internal and external forces. More specifically, we used the algorithm described in [14] since it is much less dependent than other similar techniques on the initial solution for extracting the target contour.

Two other examples are provided in Figure 5 for the case of the Rafale aircraft, at $\beta=0^{\circ}$ and $\beta=80^{\circ}$. The extracted shape and scattering centers are now superimposed on the reconstructed image in order to give a better insight 


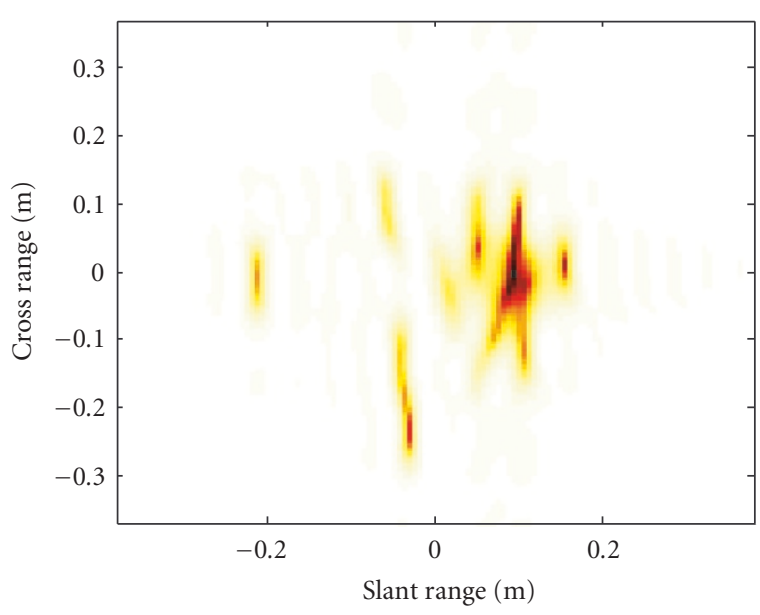

(a) Rough reconstructed image

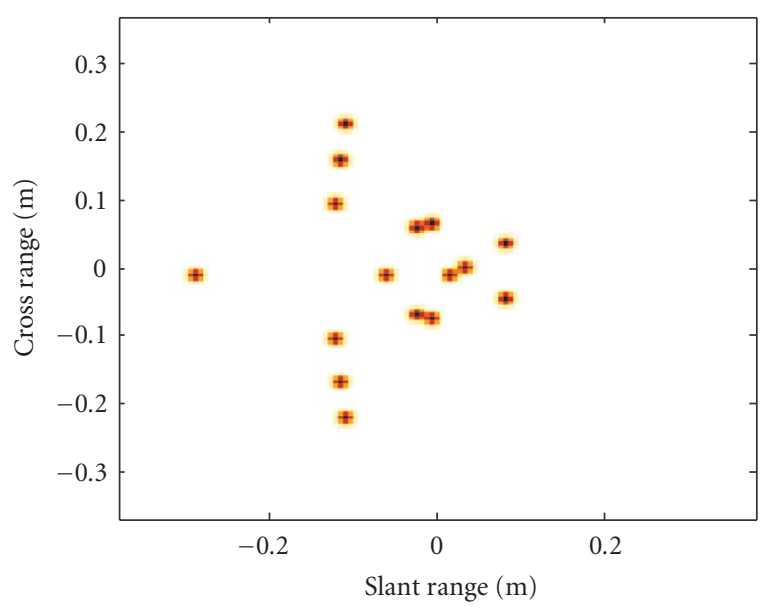

(c) Peak extraction

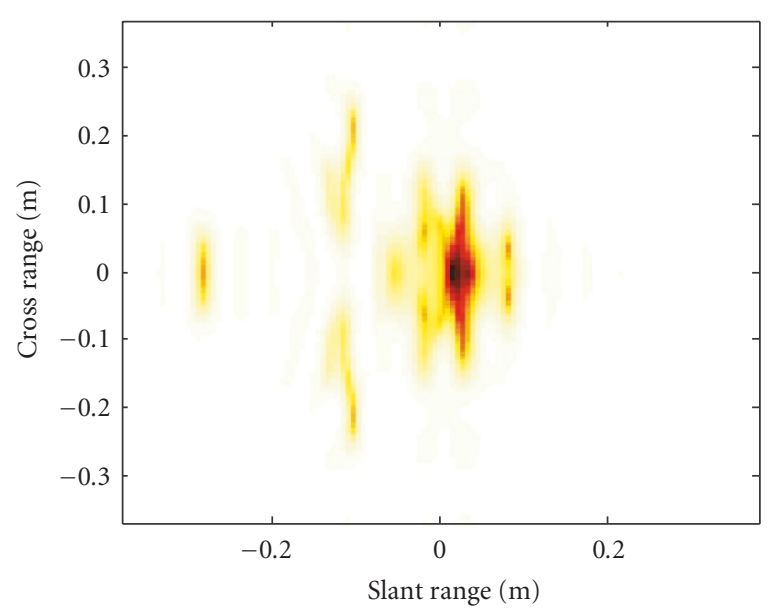

(b) Reconstructed image after phase correction

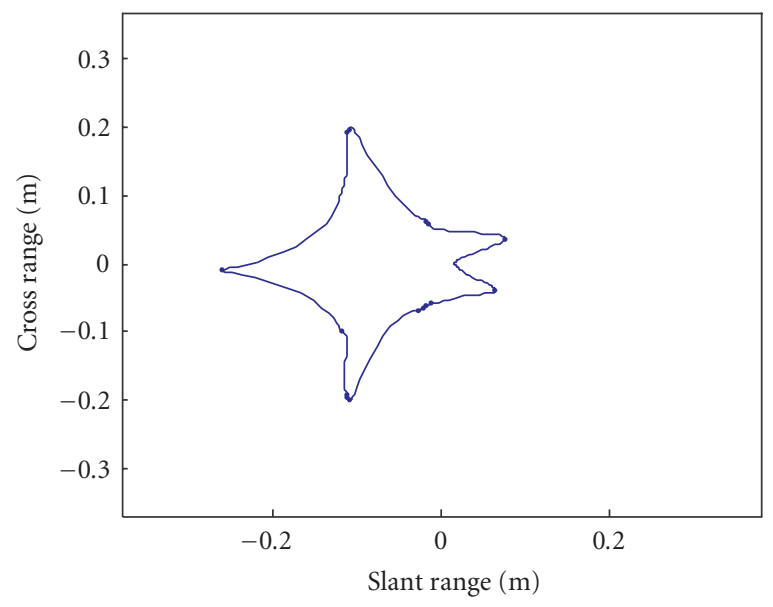

(d) Contour extraction

Figure 4: Example of contour and scattering center extraction (DC-3, $\left.\beta=0^{\circ}\right)$.

concerning the information provided by the two types of features.

Finally, some more examples of scattering center extraction are shown in Figure 6. The aspect angle is varied uniformly from $10^{\circ}$ to $90^{\circ}$ and for each angular position the scattering center distribution obtained for a different target is represented. It is thus possible to have a general, though not complete, image of the scattering center distribution characterizing each target without exhaustive use of graphical representations.

The target shape and scattering center distribution extracted as shown above cannot be used directly for feeding the classifier. Because of little a priori knowledge about target orientation, the feature vector should be rotation and shift invariant. We propose such a feature vector combining Fourier descriptors calculated from the target shape and moment invariants evaluated from the scattering center distribution.

Indeed, Fourier descriptors [15] are invariant to the translation of the shape and are not affected by rotations or a different starting point. Let $\gamma(l)=x(l)+j y(l)$ define the curve of length $L$ describing the target shape. The corresponding Fourier descriptors are then computed using the following relationship:

$F D(n)$

$$
=\frac{L}{(2 \pi n)^{2}} \sum_{k=1}^{m} b(k-1)\left(e^{-j(2 \pi / L) n l(k)}-e^{-j(2 \pi / L) n l(k-1)}\right),
$$

where $l(k)=\sum_{i=1}^{k}|\gamma(i)-\gamma(i-1)|$, with $l(0)=0$, and $b(k)=$ $(\gamma(k+1)-\gamma(k)) /|\gamma(k+1)-\gamma(k)|$.

Only the first 5 Fourier descriptors have been included in the feature vector. Increasing their number makes the feature vector more sensitive to noise without a significant improvement of its discriminant capability.

Just like the Fourier descriptors, the moment invariants do not depend on the target translation or rotation. Zernike moment-based invariants [16] or moment invariants introduced by $\mathrm{Hu}$ [17] have been the most widely used so far. In 


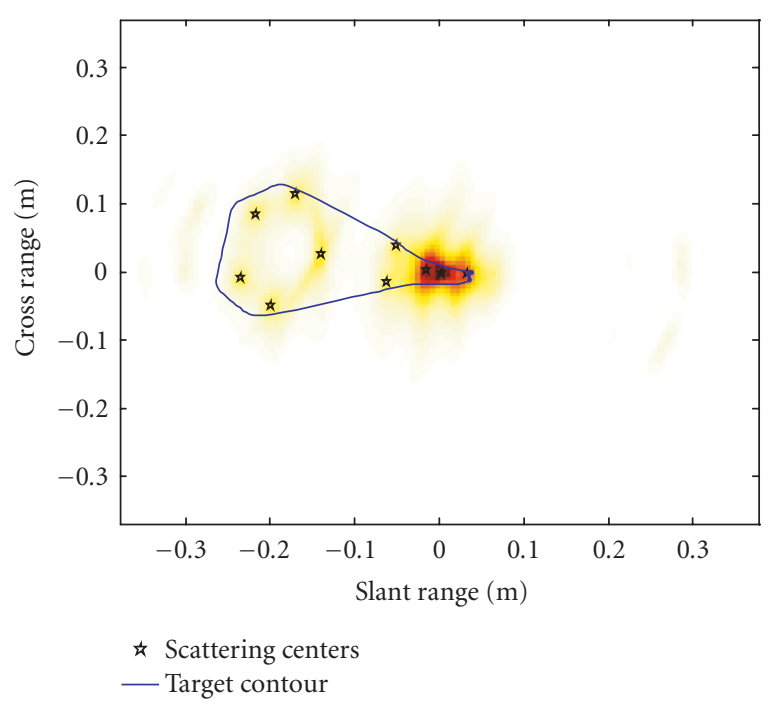

(a)

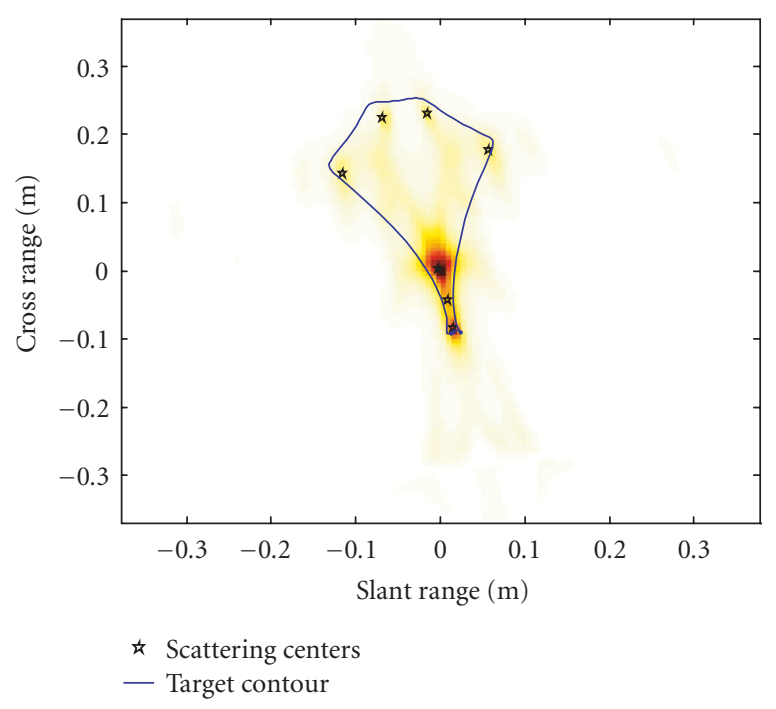

(b)

FIgURE 5: Examples of contour and scattering center extraction: (a) Rafale, $\beta=10^{\circ}$, (b) Rafale, $\beta=80^{\circ}$.

our experiment we have used the three-dimensional moment invariants defined in [18], which have shown both good discriminant capability and noise robustness:

$$
\begin{aligned}
J_{1}= & \mu_{200}+\mu_{020}+\mu_{002}, \\
J_{2}= & \mu_{200} \mu_{020}+\mu_{200} \mu_{002}+\mu_{020} \mu_{002}-\mu_{110}^{2}-\mu_{101}^{2}-\mu_{011}^{2}, \\
J_{3}= & \mu_{200} \mu_{020} \mu_{002}+2 \mu_{110} \mu_{101} \mu_{011}-\mu_{002} \mu_{110}^{2} \\
& -\mu_{020} \mu_{101}^{2}-\mu_{200} \mu_{011}^{2},
\end{aligned}
$$

where

$$
\mu_{p q r}=\sum_{m=1}^{N_{\mathrm{sc}}} \Psi\left(x_{m}, y_{m}, z_{m}\right)\left(x_{m}-\bar{x}\right)^{p}\left(y_{m}-\bar{y}\right)^{q}\left(z_{m}-\bar{z}\right)^{r} .
$$

$N_{\mathrm{sc}}$ stands for the number of scattering centers, $(\bar{x}, \bar{y}, \bar{z})$ represents the target centroid, while $\Psi\left(x_{m}, y_{m}, z_{m}\right)$ is the intensity of the $m$ th scattering center.

Actually, our scattering center distributions are bidimensional. So, $J_{3}=0$, and only $J_{1}$ and $J_{2}$ are added to the feature vector, which has 7 components in its final form. An example is provided in Figure 7 to illustrate the rotation and shift invariance of the feature vector.

\section{SART CLASSIFIER}

ART is basically a class of clustering methods. A clustering algorithm maps a set of input vectors to a set of clusters according to a specific similarity measure. Clusters are usually internally represented using prototype vectors. A prototype is typical of a group of similar input vectors defining a cluster. Both the clusters and the associated prototypes are obtained using a specific learning or training algorithm.
All classifiers are subject to the so-called stability-plasticity dilemma [19]. A training algorithm is plastic if it retains the potential to adapt to new input vectors indefinitely and it is stable if it preserves previously learned knowledge.

Consider, for instance, the case of a backpropagation neural network. The weights associated with the network neurons reach stable values at the end of the training process which is aimed to minimize the learning error and to maximize the generalization capability. The number of required neurons is minimized because all of them pull together to form the separating surface between each couple of classes. However, the classification accuracy of those types of neural networks will rapidly decrease whenever the input environment changes. In order to remain plastic, the network has to be retrained. If just the new input vectors are used in this phase, the old information is lost and the classification accuracy evaluated on the old input vectors will rapidly decrease again. So, the algorithm is not stable and the only solution is to retrain the network using each time the entire database. It is obviously not a practical solution since the computation burden increases significantly.

ART was conceived to provide a suitable solution to the stability-plasticity dilemma [20]. Two unsupervised ART neural networks were first designed: ART-1 [19] for binary input vectors and ART-2 [21] for continuous ones as well. Several adaptations have then been proposed: ART3 [22], ART-2a [23], ARTMAP [24], fuzzy ART [25], and fuzzy ARTMAP [26]. Some other unsupervised neural networks have also been inspired by ART principle such as SMART (self-consistent modular ART) [27], HART (hierarchical ART) [28], or CALM (categorizing and learning model) [29].

SART (supervised ART) [30] is a classifier similar to ART neural networks, but it is designed to operate in a supervised framework. It has the capability to learn quickly using local 


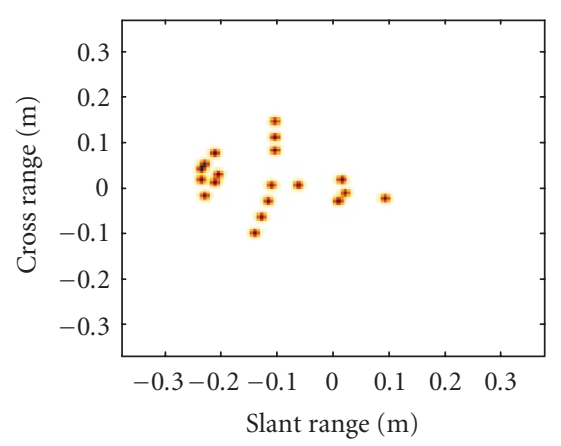

(a) F-14, $\beta=10^{\circ}$

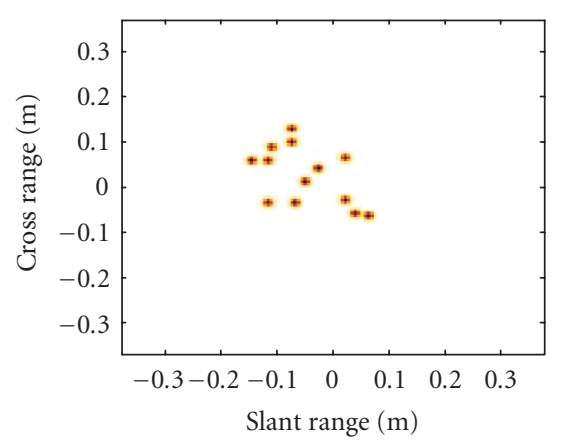

(d) F-16, $\beta=40^{\circ}$

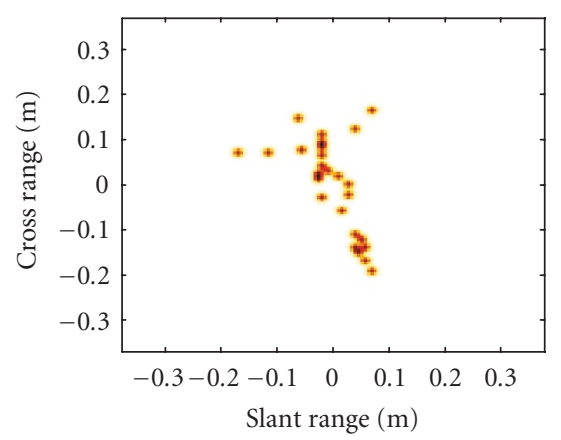

(g) Tornado, $\beta=70^{\circ}$

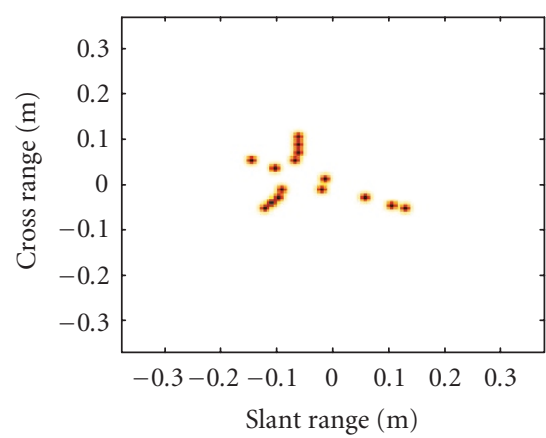

(b) Jaguar, $\beta=20^{\circ}$

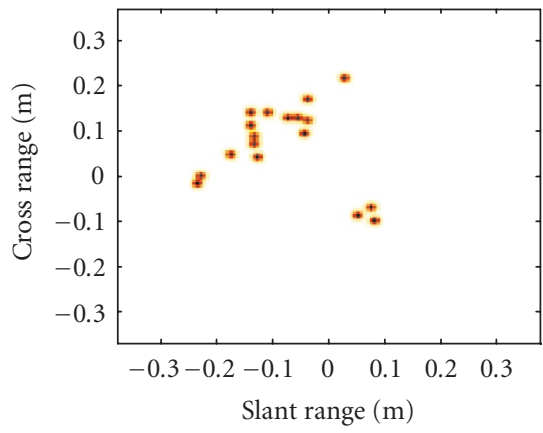

(e) F-117, $\beta=50^{\circ}$

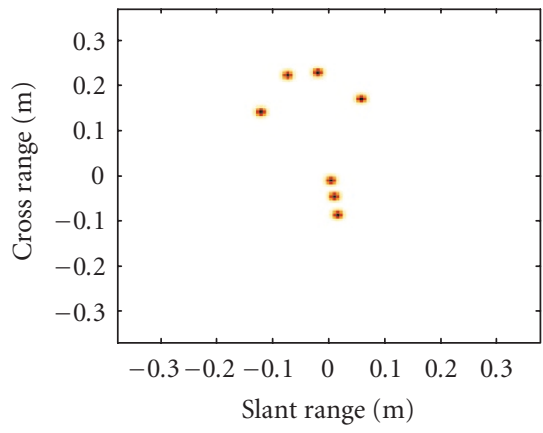

(h) Rafale, $\beta=80^{\circ}$

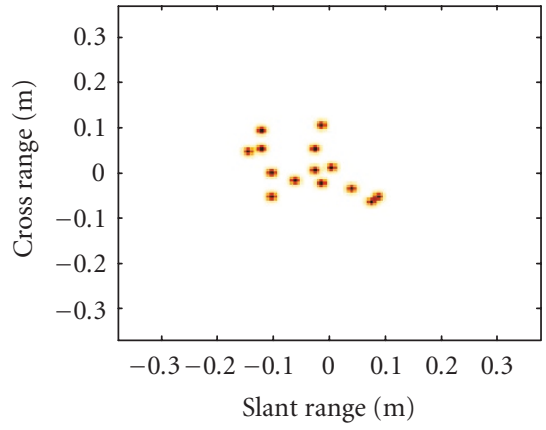

(c) Mirage, $\beta=30^{\circ}$

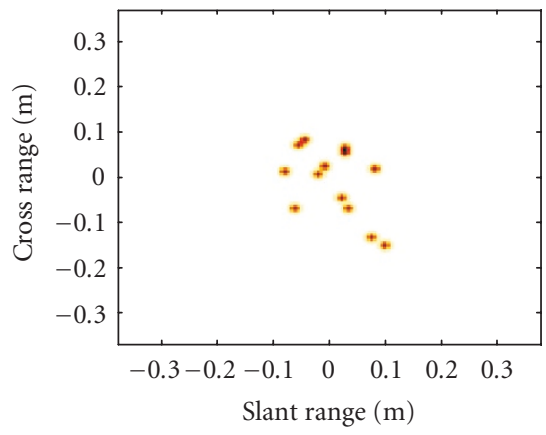

(f) Harrier, $\beta=60^{\circ}$

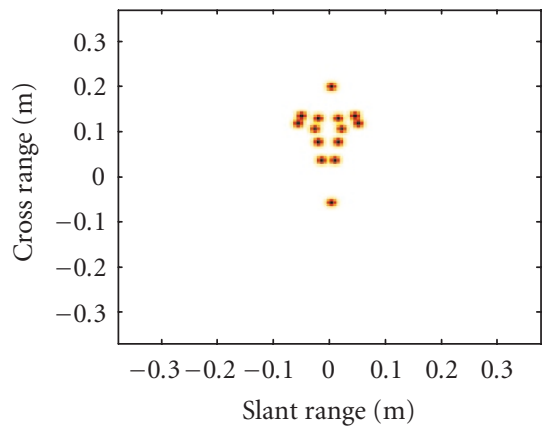

(i) $\mathrm{AH}-64, \beta=90^{\circ}$

FIgURE 6: Examples of superresolution images after scattering center extraction.

approximations of each class distribution and its operation does not depend on any chosen parameter. A prototype set is first dynamically created and modified according to the algorithm that will be described below. It is very similar to the $Q^{*}$-algorithm [31], but provides a better generalization capability.

Let us denote by $\mathbf{x}_{k}^{(j)}$ the $k$ th input vector belonging to the class $j$ and $\mathbf{p}_{k}^{(j)}$ the $k$ th prototype associated to this class. Each class $C_{j}=\left\{\mathbf{x}_{k}^{(j)}\right\}_{k=1, \ldots, N_{j}}$ is represented by one or several prototypes $\left\{\mathbf{p}_{k}^{(j)}\right\}_{k=1, \ldots, P_{j}}$ which approximate the modes of the underlying probability density function, with $N_{j}$ and $P_{j}$ being the number of vectors and of the prototypes corresponding to the class $j$. These prototypes play the same role as the codebook vectors for an LVQ (learning vector quantization) neural network [32] or the hidden layer weight vectors for an RBF (radial basis function) neural network [33].

The training algorithm starts by randomly setting one prototype for each class. The basic idea is to create a new prototype for a class whenever the actual set of prototypes is no longer able to classify the training data set satisfactorily using the nearest prototype rule:

$$
\left\|\mathbf{x}-\mathbf{p}_{l}^{(i)}\right\|=\min _{j=1, \ldots, M, k=1, \ldots, N_{j}}\left\|\mathbf{x}-\mathbf{p}_{k}^{(j)}\right\| \Longrightarrow \mathbf{x} \in C_{i}
$$

If, for example, the vector $\mathbf{x}$ previously classified does not actually belong to the class $C_{i}$, but to another class, say $C_{r}$, 


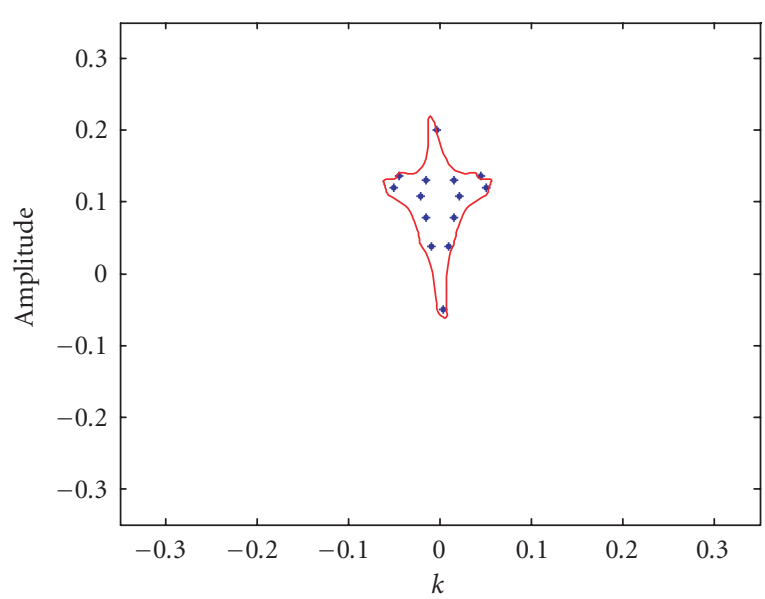

* Scattering centers

- Target contour

(a) F-14, $\beta=10^{\circ}$

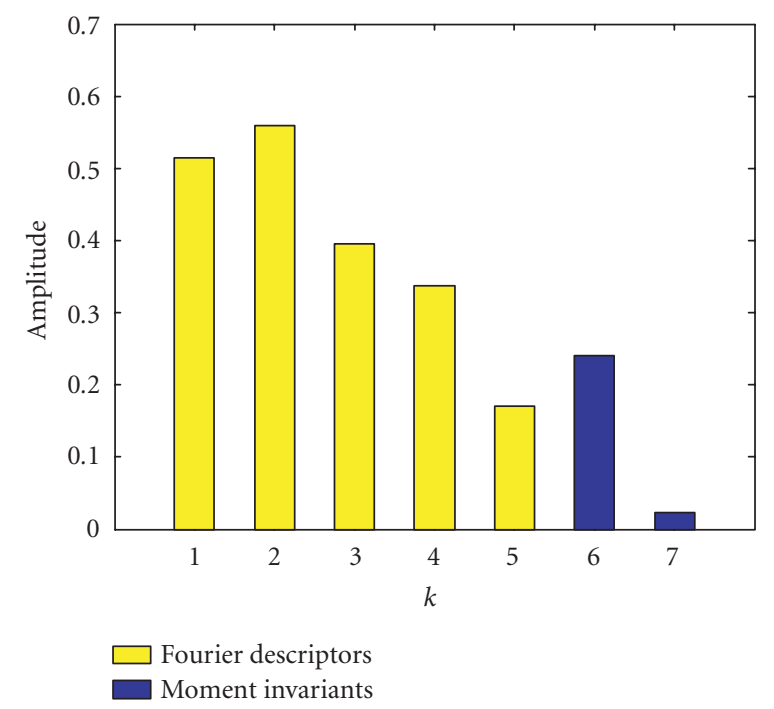

(c) F- $14, \beta=10^{\circ}$

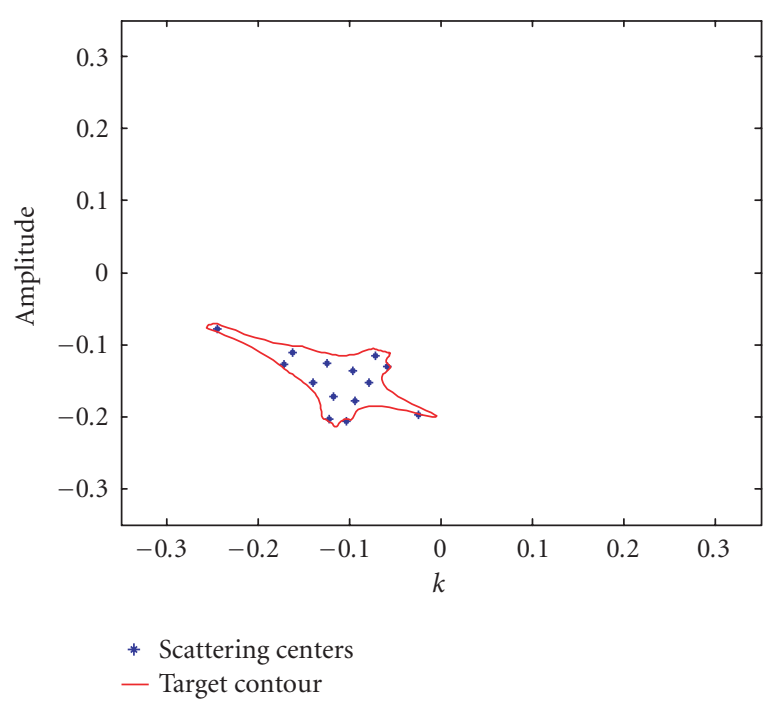

(b) F-14, $\beta=10^{\circ}$

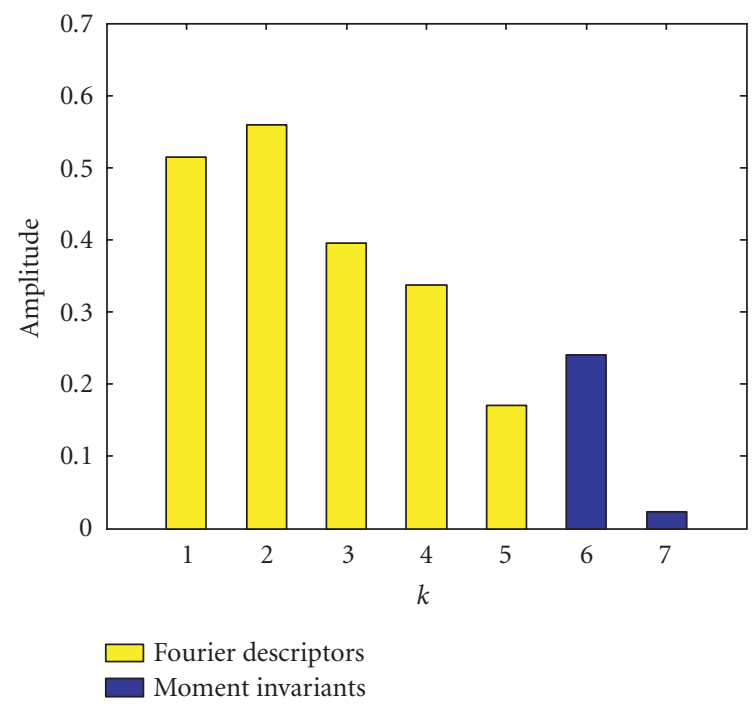

(d) F-14, $\beta=10^{\circ}$

FIGURE 7: Rotation and shift invariance of the feature vector-example for AH-64 at (a,c) $\beta=90^{\circ}$ and (b,d) $\beta=10^{\circ}$. (a) Original target. (b) Shifted and rotated target. (c) Feature vector extracted from the original target. (d) Feature vector extracted from the shifted and rotated target.

then a new prototype $\mathbf{p}_{N_{r}+1}^{(r)}=\mathbf{x}$ will be added to the list of prototypes of the class $C_{r}$.

Let card denote the cardinal number of a given set. Consider $A_{l}^{(i)}$ the input vector set well classified with respect to the prototype $\mathbf{p}_{l}^{(i)}$. The prototypes are updated during each epoch using the mean of the samples which are correctly classified by each of them:

$$
\mathbf{p}_{l}^{(i)}=\frac{1}{\operatorname{card}\left\{A_{l}^{(i)}\right\}} \sum_{\mathbf{x}_{m} \in A_{l}^{(i)}} \mathbf{x}_{m}
$$

with

$$
A_{l}^{(i)}=\left\{\mathbf{x}_{m}^{(i)} \mid\left\|\mathbf{x}_{m}^{(i)}-\mathbf{p}_{l}^{(i)}\right\|=\min _{j=1, \ldots, M, k=1, \ldots, N_{j}}\left\|\mathbf{x}_{m}^{(i)}-\mathbf{p}_{k}^{(j)}\right\|\right\} .
$$

A prototype is cancelled if it does not account for a minimum number of well-classified training vectors because this suggests it is unduly influenced by outliers:

$$
\operatorname{card}\left\{A_{l}^{(i)}\right\} \leq N_{t} \Longrightarrow \mathbf{p}_{l}^{(i)} \text { is cancelled. }
$$

This iterative learning process continues as long as the number and the location of the prototypes change. The corresponding flowchart is shown on the left side of Figure 8. 


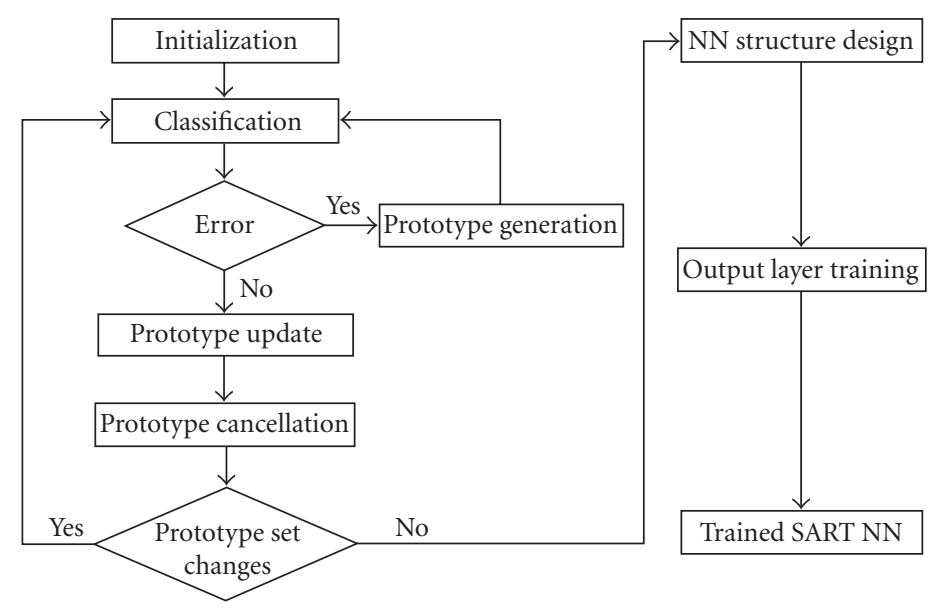

FIGURE 8: SART learning process flowchart.

An important property of the described algorithm is that it needs no initial system parameter specifications and no prespecified number of codebook or center vectors. Indeed, unlike the RBF or LVQ neural network, the number and the final values of the prototypes are automatically found during the training process for the SART classifier.

The prototypes calculated in this way will be the weight vectors of the hidden layer neurons of SART as indicated in Figure 9. So, the number of the neurons on the hidden layer is equal to the number of prototypes, denoted by $L$ in this figure.

Each hidden neuron computes the distance between the test vector $\mathbf{x}$ and the associated prototype. This distance is then normalized in order to take into account the different spreads of the clusters represented by the prototypes:

$$
\tilde{d}_{k}=\frac{d_{k}}{d_{k \max }}=\frac{\left\|\mathbf{x}-\mathbf{p}_{k}\right\|}{d_{k \max }},
$$

where

$$
d_{k \max }=\max _{\mathbf{x}_{i} \in A_{k}}\left\|\mathbf{x}_{i}-\mathbf{p}_{k}\right\| .
$$

The outputs of the neurons from the hidden layer are finally calculated using the following relationship:

$$
y_{k}=f\left(\tilde{d}_{k}\right)=\left(1+\tilde{d}_{k}^{2}\right)^{-1} .
$$

The activation function $f$ is close to a Gaussian one, but is easier to compute. While its value can vary between 0 and 1 only the input vectors belonging to the neuron's cluster are able to produce values above 0.5 . Indeed, it can be readily seen that at the cluster boundaries the activation function equals 0.5:

$$
\left.f\left(\tilde{d}_{k}\right)\right|_{d_{k}=d_{k \max }}=f(1)=0.5 .
$$

Hence, $f$ can also be seen as a cluster membership function since its value clearly indicates whether an input vector is inside or outside the cluster.
The output layer of SART is a particular type of linear neural network, called MADALINE (multiple adaptive linear network) [32]. It is aimed at combining the hidden layer outputs $\left\{y_{k}\right\}_{k=1, \ldots, L}$, such that only one output neuron represents each class. Let $t_{m}$ and $o_{m}$ denote the target and real outputs for the $m$ th neuron of this layer. The Widrow-Hoff rule [34] used to train this layer can then be expressed in the following form:

$$
\begin{aligned}
\Delta w_{m k} & =\eta\left(t_{m}-o_{m}\right) y_{k}, \\
\Delta b_{m} & =\eta\left(t_{m}-o_{m}\right),
\end{aligned}
$$

where $\left\{w_{m k}\right\}_{m=1, \ldots, M, k=1, \ldots, L}$ and $\left\{b_{m}\right\}_{m=1, \ldots, M}$ stand for the weights and biases of the neurons from the output layer, $M$ is the number of classes, and $\eta$ is the learning rate.

\section{CLASSIFICATION RESULTS}

A database containing 900 feature vectors has been generated by applying the approach described in Section 3 to the superresolution images of the ten targets, reconstructed as indicated in Section 2. SART classifier has then been used to classify them. Two other classifiers have also been used for comparison: a multilayer perceptron (MLP) [33] and a fuzzy KNN ( $K$ nearest neighbor) classifier [35].

The results reported here have been obtained using the LOO (leave one out) [36] performance estimation technique, which provides an almost unbiased estimate of the classification accuracy. According to this method, at each step all the input vectors are included in the training set, except for one. It serves to test the classifier when its training is finished. This procedure is repeated so that each input vector plays once and exclusively the role of the test set. The classifier will be roughly the same each time since there is little difference between two training sets. At the end of the training process the confusion matrix is directly obtained from these partial results. 


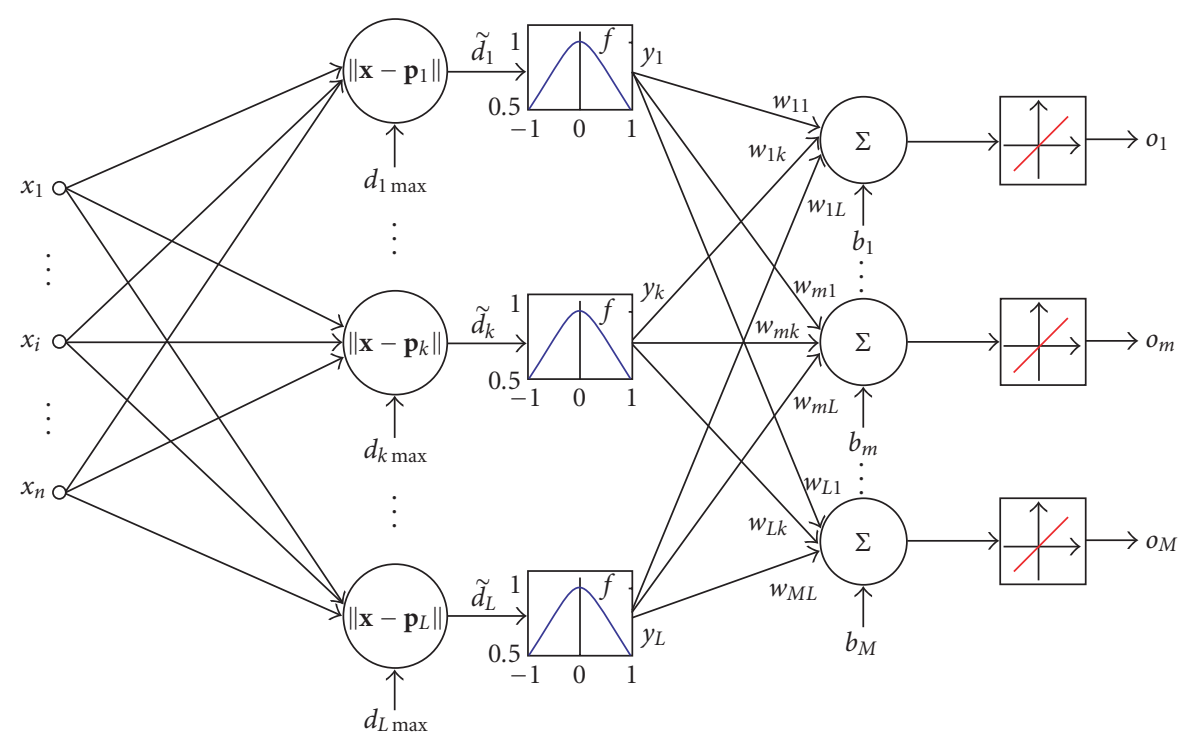

FIGURE 9: SART neural network structure.

TABle 1: Confusion matrix for MLP classifier.

\begin{tabular}{|c|c|c|c|c|c|c|c|c|c|c|c|}
\hline & \multicolumn{11}{|c|}{ Output class } \\
\hline \multirow{11}{*}{ Input class } & & Mirage & Tornado & Rafale & F-16 & DC-3 & F-14 & Harrier & Jaguar & F-117 & AH-64 \\
\hline & Mirage & 81 & 1 & 2 & 2 & 0 & 0 & 2 & 1 & 0 & 1 \\
\hline & Tornado & 1 & 83 & 1 & 2 & 0 & 2 & 1 & 0 & 0 & 0 \\
\hline & Rafale & 3 & 0 & 82 & 2 & 0 & 0 & 1 & 1 & 0 & 1 \\
\hline & F-16 & 1 & 1 & 3 & 82 & 0 & 0 & 2 & 1 & 0 & 0 \\
\hline & DC-3 & 0 & 2 & 0 & 0 & 85 & 0 & 0 & 0 & 3 & 0 \\
\hline & F-14 & 0 & 2 & 0 & 1 & 1 & 84 & 0 & 0 & 2 & 0 \\
\hline & Harrier & 2 & 0 & 1 & 2 & 0 & 0 & 83 & 2 & 0 & 0 \\
\hline & Jaguar & 1 & 0 & 1 & 1 & 0 & 0 & 3 & 83 & 0 & 1 \\
\hline & F-117 & 0 & 1 & 0 & 0 & 2 & 2 & 0 & 0 & 85 & 0 \\
\hline & AH-64 & 1 & 0 & 0 & 1 & 0 & 0 & 0 & 2 & 0 & 86 \\
\hline
\end{tabular}

The training parameters for the 3 classifiers have been chosen to maximize the mean classification rate. For the fuzzy KNN classifier the training stage is equivalent to a fuzzyfication procedure, where the membership coefficients for each class are calculated for all the training vectors.

Let $V_{K}(\mathbf{x})$ be the $K$ th order neighborhood of the vector $\mathbf{x}$. We have used the following relationship to calculate the membership coefficient of the training vector $\mathbf{x}_{l}$ for the class $C_{j}$ :

$$
u_{j l}=\frac{K_{j}^{(l)}}{K_{F}}, \quad K_{j}^{(l)}=\operatorname{card}\left\{\mathbf{x}_{n}^{(j)} \mid \mathbf{x}_{n}^{(j)} \in V_{K_{F}}\left(\mathbf{x}_{l}\right)\right\} .
$$

In the equation above $\mathbf{x}_{n}^{(j)}$ is the $n$th training vector of the class $C_{j}, K_{F}$ defines the neighborhood value during the training stage, while $K_{j}^{(l)}$ stands for the number of the nearest neighbors of the vector $\mathbf{x}_{l}$ belonging to the class $C_{j}$. In our experiment we have used $K_{F}=15$. The same number of nearest neighbors has also been considered to make the decision in the classification phase.
The training process of SART has resulted with an optimum number of 47 prototypes. Consequently, SART neural network has been designed with 47 neurons on the hidden layer and 10 neurons on the output layer. A learning rate $\eta=0.1$ has been set for the output layer.

The same number of layers and neurons are considered for the MLP. The activation function for both the hidden and the output layer is of log-sigmoid type. MLP is trained using the gradient descent with momentum and adaptive learning rate backpropagation algorithm. The learning rate reference value is 0.01 , while the momentum constant is 0.9 .

The classification results obtained with the 3 classifiers are presented on Tables 1 to 4 . Tables 1 to 3 give the confusion matrix for each classifier. The $k$ th diagonal element of such a matrix indicates the number of images that are correctly classified for the class $k$. Any other element, corresponding, for example, to the row $k$ and the column $j$, gives the number of images from the class $k$, which are classified in the class $j$. Note that the sum of the elements of the row $k$ equals the number of images belonging to the class $k$ (recall that each 
TABLE 2: Confusion matrix for fuzzy-KNN classifier.

\begin{tabular}{|c|c|c|c|c|c|c|c|c|c|c|c|}
\hline & \multicolumn{11}{|c|}{ Output class } \\
\hline \multirow{11}{*}{ Input class } & & Mirage & Tornado & Rafale & F-16 & DC-3 & F-14 & Harrier & Jaguar & F-117 & AH-64 \\
\hline & Mirage & 80 & 1 & 1 & 3 & 0 & 0 & 2 & 3 & 0 & 0 \\
\hline & Tornado & 1 & 86 & 1 & 0 & 0 & 2 & 0 & 0 & 0 & 0 \\
\hline & Rafale & 2 & 0 & 82 & 3 & 0 & 0 & 1 & 2 & 0 & 0 \\
\hline & F-16 & 3 & 1 & 4 & 81 & 0 & 0 & 0 & 1 & 0 & 0 \\
\hline & DC-3 & 0 & 0 & 0 & 0 & 88 & 0 & 0 & 0 & 2 & 0 \\
\hline & F-14 & 0 & 1 & 0 & 0 & 0 & 88 & 0 & 0 & 1 & 0 \\
\hline & Harrier & 2 & 0 & 0 & 0 & 0 & 0 & 85 & 3 & 0 & 0 \\
\hline & Jaguar & 2 & 0 & 0 & 1 & 0 & 0 & 2 & 84 & 0 & 1 \\
\hline & F-117 & 0 & 2 & 0 & 0 & 1 & 2 & 0 & 0 & 85 & 0 \\
\hline & AH-64 & 0 & 0 & 0 & 1 & 0 & 0 & 0 & 2 & 0 & 87 \\
\hline
\end{tabular}

TABLE 3: Confusion matrix for SART classifier.

\begin{tabular}{|c|c|c|c|c|c|c|c|c|c|c|c|}
\hline & \multicolumn{11}{|c|}{ Output class } \\
\hline \multirow{11}{*}{ Input class } & & Mirage & Tornado & Rafale & F-16 & DC-3 & F-14 & Harrier & Jaguar & F-117 & $\mathrm{AH}-64$ \\
\hline & Mirage & 81 & 1 & 2 & 1 & 0 & 0 & 3 & 2 & 0 & 0 \\
\hline & Tornado & 1 & 85 & 1 & 0 & 0 & 2 & 0 & 0 & 1 & 0 \\
\hline & Rafale & 2 & 0 & 82 & 3 & 0 & 0 & 1 & 2 & 0 & 0 \\
\hline & F-16 & 3 & 1 & 2 & 83 & 0 & 0 & 0 & 1 & 0 & 0 \\
\hline & DC-3 & 0 & 0 & 0 & 0 & 87 & 1 & 0 & 0 & 2 & 0 \\
\hline & F-14 & 0 & 1 & 0 & 0 & 0 & 88 & 0 & 0 & 1 & 0 \\
\hline & Harrier & 1 & 0 & 1 & 1 & 0 & 0 & 85 & 2 & 0 & 0 \\
\hline & Jaguar & 1 & 0 & 1 & 1 & 0 & 0 & 2 & 85 & 0 & 0 \\
\hline & F-117 & 0 & 1 & 0 & 0 & 2 & 1 & 0 & 0 & 86 & 0 \\
\hline & AH-64 & 1 & 0 & 0 & 1 & 0 & 0 & 0 & 2 & 0 & 86 \\
\hline
\end{tabular}

TABle 4: Mean classification rate for each classifier.

\begin{tabular}{lcc}
\hline MLP & KNNF & SART \\
\hline $92.67 \%$ & $94 \%$ & $94.22 \%$ \\
\hline
\end{tabular}

class contains 90 images). The classification rate for the class $k$ is then obtained as the ratio between the $k$ th diagonal element and the sum of the elements of the row $k$. The classification rates obtained in this way for each classifier and each class are illustrated in Figure 10. Finally, the mean classification rates for the 3 classifiers are provided in Table 4 . These values are obtained by averaging the classification rates over the 10 classes.

The 3 classifiers have also been compared in terms of training and classification speed. The results indicated in Figure 11 have been obtained on a PC Pentium IV, operating at $1800 \mathrm{MHz}$. We have used 3 Gaussian classes having the same number of training and test vectors. Both training and classification times have been measured for 6 values of the training/test vectors: $100,500,1000,1500,2000$, and 2500, as indicated in Figure 11.

Although the classification accuracy for SART, as summarized in Table 4, is slightly better than that for the two other classifiers tested, the performances of all three classifiers are similar. Nevertheless, thanks to its neural network structure, SART classification times are very close to those obtained for the MLP and much shorter than those obtained for the fuzzy KNN classifier. On the other hand, SART always learns faster than MLP and it learns faster than fuzzy KNN too, whenever the number of training vectors is large (over 1000 vectors in our experiment).

\section{CONCLUSION}

An end-to-end application is described in the paper for the supervised self-organizing classification of superresolution ISAR images. The proposed approach is suitable for the classification of radar targets measured in an anechoic chamber.

All the images are obtained using MUSIC-2D method, which is an effective and easy-to-use orthogonal subspace decomposition-based imaging technique. It is able to provide superresolution and to accurately recover the scattering center locations even for a small number of correlated samples.

The two types of features extracted from the reconstructed images, Fourier descriptors and moment invariants, ensure a good separation of imaged targets. Since these features are invariant to the target position and orientation, the database is highly compressed.

SART neural network, used for the classification stage, combines the vector quantization and the adaptive resonance theory principles, which results in a powerful selforganizing neural network structure. The particular form of 


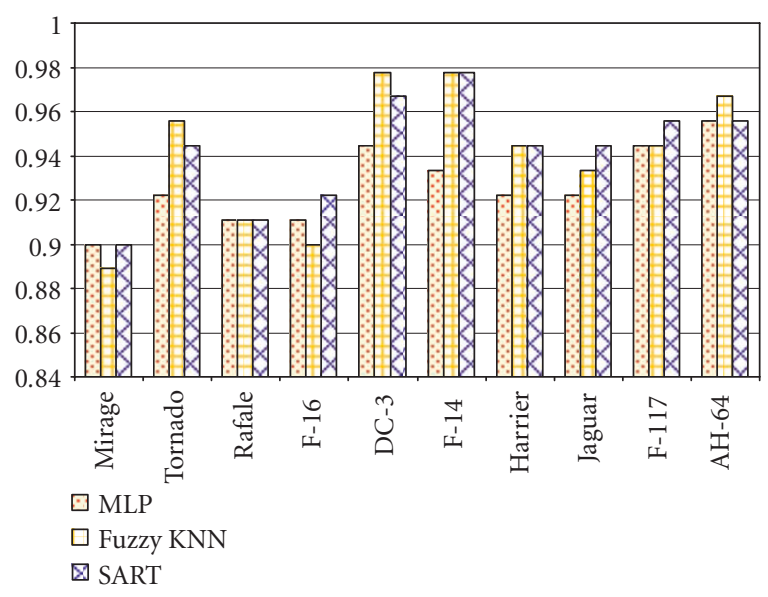

Figure 10: Mean classification rates for each target and each classifier.

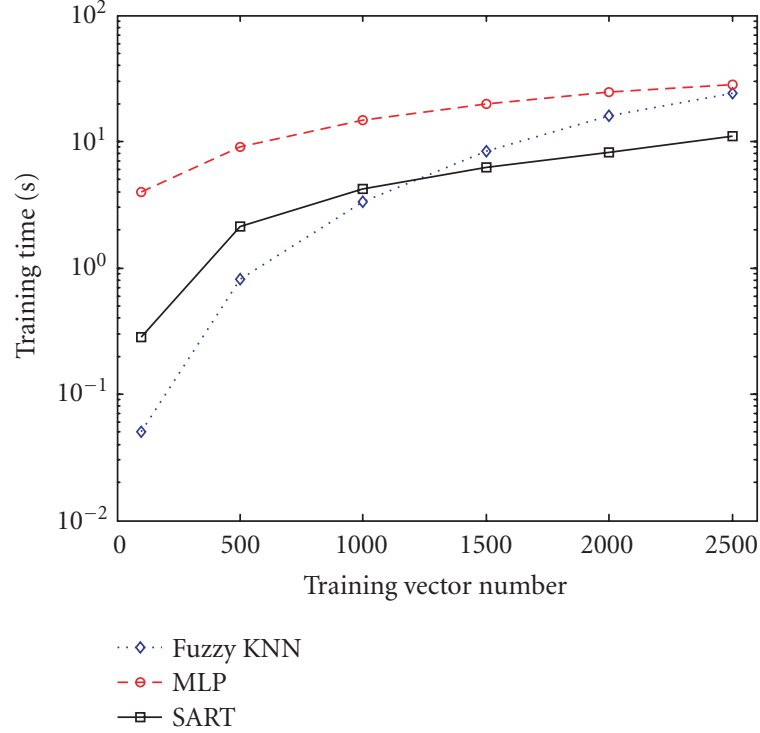

(a) $\mathrm{F}-14, \beta=10^{\circ}$

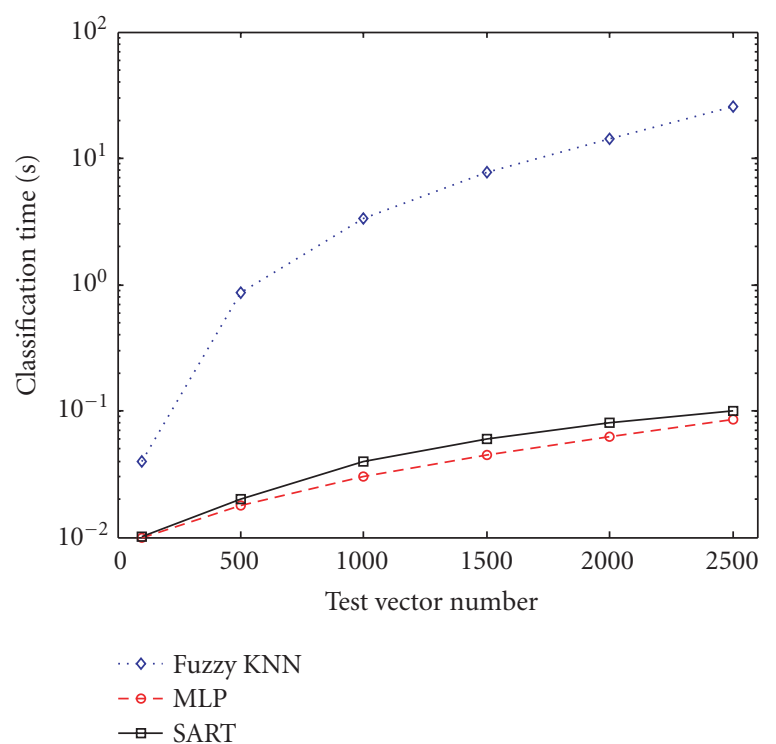

(b) $\mathrm{F}-14, \beta=10^{\circ}$

FIGURE 11: Training and classification speeds for fuzzy KNN, MLP, and SART classifiers.

the threshold function and the supervised procedure used to determine the prototypes and the associated clusters allow for an adaptive partition of the input space vectors. This results in a fast and fully automatic training process thus providing high classification rate, good generalization capability, and reinforced protection against outliers. Furthermore, it is effective and easy to use because it requires only a few input parameters whose values are not critical for its performance. SART has been compared to a multilayer perceptron and to a fuzzy KNN classifier. While the mean classification rates provided by the 3 classifiers are close, SART learns more rapidly than MLP and classifies much faster than Fuzzy KNN.

For future work we intend to address the classification problem described in this paper when the pitch and roll mo- tion components of the radar target are also considered. The basic idea would be to train the classifier with a vector set issued from a discretization of the whole three dimensional angular domain rather than for azimuth aspects only. In this way the recognition system should be able to classify the targets irrespective of their orientation in the three dimensional space. Another interesting idea would be to extend this work to higher frequencies, consistent with microwave frequencies on full-sized targets ( $S$-band or $X$-band, e.g.).

\section{ACKNOWLEDGMENT}

We would like to thank the two anonymous referees for their careful reading and valuable comments and suggestions. 


\section{REFERENCES}

[1] A. Quinquis, E. Radoi, and F.-C. Totir, "Some radar imagery results using superresolution techniques," IEEE Transactions on Antennas and Propagation, vol. 52, no. 5, pp. 1230-1244, 4004.

[2] E. Radoi and A. Quinquis, "A new method for estimating the number of harmonic components in noise with application in high resolution radar," EURASIP Journal on Applied Signal Processing, vol. 2004, no. 8, pp. 1177-1188, 2004.

[3] V. G. Nebabin, Methods and Techniques of Radar Recognition, Artech House, London, UK, 1994.

[4] R. Wehner, High Resolution Radar, Artech House, Boston, Mass, USA, 2nd edition, 1994.

[5] J. W. Odendaal, E. Barnard, and C. W. I. Pistorius, "Twodimensional superresolution radar imaging using the MUSIC algorithm," IEEE Transactions on Antennas and Propagation, vol. 42, no. 10, pp. 1386-1391, 1994.

[6] P. Stoica and T. Söderström, "Statistical analysis of MUSIC and subspace rotation estimates of sinusoidal frequencies," IEEE Transactions on Signal Processing, vol. 39, no. 8, pp. 1836-1847, 1991.

[7] A. Gersho and R. M. Gray, Vector Quantization and Signal Compression, Kluwer Academic Press/Springer, Boston, Mass, USA, 1992.

[8] G. A. Carpenter and S. Grossberg, Pattern Recognition by SelfOrganizing Neural Networks, MIT Press, Cambridge, Mass, USA, 1991.

[9] T.-J. Shan, M. Wax, and T. Kailath, "On spatial smoothing for direction-of-arrival estimation of coherent signals," IEEE Transactions on Acoustics, Speech, and Signal Processing, vol. 33, no. 4, pp. 806-811, 1985.

[10] M. Wax and T. Kailath, "Detection of signals by information theoretic criteria," IEEE Transactions on Acoustics, Speech, and Signal Processing, vol. 33, no. 2, pp. 387-392, 1985.

[11] M. Desvignes, S. Langlois, J. M. Constans, and M. Revenu, "Phase unwrapping: geometric distortions correction on MRI," Traitement du Signal, vol. 17, no. 4, pp. 313-324, 2000.

[12] Y. Wu and D. C. Munson Jr., "Wide-angle ISAR passive imaging using smoothed pseudo Wigner-Ville distribution," in Proceedings of the IEEE Radar Conference, pp. 363-368, Atlanta, Ga, USA, May 2001.

[13] M. Kass, A. Witkin, and D. Terzopoulos, "Snakes: active contour models," International Journal of Computer Vision, vol. 1, no. 4, pp. 321-331, 1987.

[14] C. Xu and J. L. Prince, "Snakes, shapes and gradient vector flow," IEEE Transactions on Image Processing, vol. 7, no. 3, pp. 359-369, 1998.

[15] E. Persoon and K. S. Fu, "Shape discrimination using Fourier descriptors," IEEE Transactions on Systems, Man, and Cybernetics, vol. 7, no. 3, pp. 171-179, 1977.

[16] M. R. Teague, "Image analysis via the general theory of moments," Journal of the Optical Society of America, vol. 70, no. 8, pp. 920-930, 1980.

[17] M. K. Hu, "Visual pattern recognition by moment invariants," IRE Transactions on Information Theory, vol. 8, no. 2, pp. 179$187,1962$.

[18] F. A. Sadjadi and E. L. Hall, "Three-dimensional moment invariants," IEEE Transactions on Pattern Analysis and Machine Intelligence, vol. PAMI-2, no. 2, pp. 127-137, 1980.

[19] G. Carpenter and S. Grossberg, "A massively parallel architecture for a self organizing neural pattern recognition machine,"
Computer Vision, Graphics, and Image Processing, vol. 37, no. 1, pp. 54-115, 1987.

[20] S. Grossberg, "Adaptive pattern classification and universal recoding. II. Feedback, expectation, olfacation, and illusions," Biological Cybernetics, vol. 23, no. 4, pp. 187-202, 1976.

[21] G. Carpenter and S. Grossberg, "ART 2: self-organization of stable category recognition codes for analog input patterns," Applied Optics, vol. 26, no. 23, pp. 4919-4930, 1987.

[22] G. Carpenter and S. Grossberg, "ART3: hierarchical search using chemical transmitters in self-organizing pattern recognition architectures," Neural Networks, vol. 3, no. 2, pp. 129-152, 1990.

[23] G. Carpenter, S. Grossberg, and D. B. Rosen, "ART2-A: an adaptive resonance algorithm for rapid category learning and recognition," Neural Networks, vol. 4, pp. 493-504, 1991.

[24] G. Carpenter, S. Grossberg, and J. H. Reynolds, "ARTMAP: supervised real-time learning and classification of nonstationary data by a self-organizing neural network," Neural Networks, vol. 4, pp. 565-588, 1991.

[25] G. Carpenter, S. Grossberg, and D. B. Rosen, "Fuzzy ART: fast stable learning and categorization of analog patterns by an adaptive resonance system," Neural Networks, vol. 4, pp. 759$771,1991$.

[26] G. Carpenter, S. Grossberg, N. Markuzon, J. H. Reynolds, and D. B. Rosen, "Fuzzy ARTMAP: a neural network architecture for incremental supervised learning of analog multidimensional maps," IEEE Transactions on Neural Networks, vol. 3, no. 5, pp. 698-713, 1992.

[27] G. Bartfai, "Hierarchical clustering with ART neural networks," Tech. Rep. CS-TR-94/1, Department of Computer Science, Victoria University of Wellington, Wellington, New Zealand, 1994.

[28] G. Bartfai, "An ART-based modular architecture for learning hierarchical clusterings," Tech. Rep. CS-TR-95/3, Department of Computer Science, Victoria University of Wellington, Wellington, New Zealand, 1995.

[29] J. M. J. Murre, R. H. Phaf, and G. Wolters, "CALM networks: a modular approach to supervised and unsupervised learning," in Proceedings of the International Joint Conference on Neural Networks, pp. 649-665, New York, NY, USA, 1989.

[30] E. Radoi, A. Quinquis, and F. Totir, "Superresolution ISAR image classification using Fourier descriptors and SART neural network," in Proceedings of the European Conference on Synthetic Aperture Radar, Ulm, Germany, May 2004.

[31] T. W. Rauber, D. Coltuc, and A. S. Steiger-Garção, "Multivariate discretization of continuous attributes for machine learning," in Proceedings of International Symposium on Methodologies for Intelligent Systems, pp. 80-94, Trondheim, Norway, June 1993.

[32] L. Fausett, Fundamentals of Neural Networks: Architectures, Algorithms, and Applications, Prentice-Hall, Englewood Cliffs, NJ, USA, 1994.

[33] C. M. Bishop, Neural Networks for Pattern Recognition, Oxford University Press, New York, NY, USA, 1995.

[34] R. O. Duda, P. O. Hart, and D. G. Stork, Pattern Classification, John Wiley \& Sons, New York, NY, USA, 2000.

[35] J. M. Keller, R. Gray, and J. A. Givens, "A fuzzy $k$-nearest neighbor algorithm," IEEE Transactions on Systems, Man, and Cybernetics, vol. 15, no. 4, pp. 580-585, 1981.

[36] P. A. Devijver and J. Kittler, Pattern Recognition: A Statistical Approach, Prentice-Hall, London, UK, 1982. 
Emanuel Radoi graduated with a degree in radar systems from the Military Technical Academy of Bucharest, in 1992. In 1997 he received the M.S. degree in electronic engineering, and in 1999 the Ph.D. degree in signal processing, both from the University of Brest. From 1992 to 2002, he taught and developed research activities in the radar systems field at the Military Technical Academy of Bucharest. In 2003 he

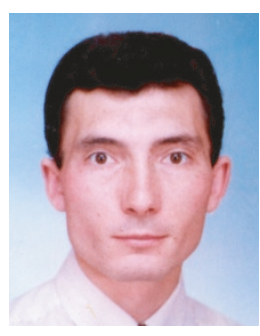
joined the Engineering School ENSIETA, Brest, where he is currently an Associate Professor. His main research interests include superresolution methods, radar imagery, automatic target recognition, and information fusion.

André Quinquis received the M.S. degree in 1986 and the Ph.D. degree in 1989, in signal processing, both from the University of Brest. From 1989 to 1992 he taught and developed research activities in signal and image processing at the Naval Academy, Brest. In 1992 he joined the Engineering School ENSIETA, Brest, where he held the positions of Senior Researcher and Head of the Electronics and Informatics Depart-

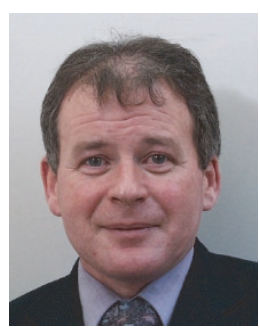
ment. Since 2001 he has been the Scientific Director of ENSIETA. $\mathrm{He}$ is mainly interested in signal processing, time-frequency methods, statistical estimation, and decision theory. He is the author of 8 books and of more than 80 papers (international journals and conferences) in the area of signal processing.

Felix Totir received the B.S. degree from Military Technical Academy of Bucharest in 2002, and the M.S. degree from the University of Brest in 2003, both in electronic engineering. He is currently with the Romanian Military Equipment and Technologies Research Agency, Bucharest. Since 2003 he has been preparing a Ph.D. thesis at ENSIETA, Brest, France. He is interested in signal processing, radar imagery, time-frequency

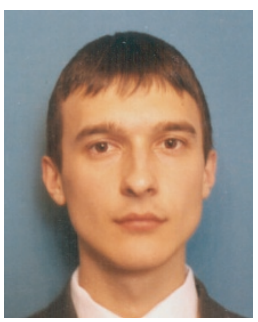
techniques, superresolution methods, and classification systems. 